'Sección Inmunología, VIH y Alergias, Hospital Clínico Universidad de Chile. Santiago, Chile.

${ }^{2}$ Sección Endocrinología, Hospital Clínico Universidad de Chile. Santiago, Chile.

${ }^{3}$ Unidad de Pacientes Críticos, Hospital Clínico Universidad de Chile. Santiago, Chile. ${ }^{4}$ Programa Formación Especialistas en Inmunología, Hospital Clínico Universidad de Chile. Santiago, Chile. ${ }^{5}$ Servicio Alergología, Hospital Universitario Ramón y Cajal, Madrid, España.

Fuentes de financiamiento: no hay. Los autores declaran no tener conflictos de interés.

Recibido el 25 de noviembre de 2017, aceptado el 26 de abril de 2018.

Correspondencia a: Dra. María Antonieta Guzmán Meléndez Sección Inmunología, VIH y Alergias, Hospital Clínico Universidad de Chile. Santiago, Chile. mguzman@hcuch.cl

\section{Desensibilización a levotiroxina. Caso clínico}

\author{
MARÍA ANTONIETA GUZMÁN ${ }^{1}$, CECILIA SEPÚLVEDA ${ }^{1}$, \\ CLAUDIO LIBERMAN ${ }^{2}$, RODRIGO CORNEJO ${ }^{3}$, \\ GIGIA ROIZEN ${ }^{4}$, DANIELA CERECEDA ${ }^{4}$, \\ MARÍA FERNANDA LARA ${ }^{4}$, EMILIO ÁLVAREZ-CUESTA ${ }^{5}$
}

\section{Successful oral desensitization to levothyroxine. Report of one case}

We report a 39-year-old female who underwent a total thyroidectomy as treatment for a thyroid papillary cancer. She suffered several episodes of mild angioedema in lips and tongue, after using different commercial Levothyroxine formulations, with and without excipients. Given the need to use this drug, the patient was admitted in our hospital and we proceeded to desensitize her with oral Levothyroxine. The patient fasted throughout the whole procedure, was properly monitored and had an adequate peripheral venous access. On the first day of the procedure, a 15-step protocol was performed, first administering placebo and then, compounded formulations of Levothyroxine starting from $0.01 \mathrm{ug}$, followed by doubling doses every 15 minutes until the cumulative dose of $111.95 \mathrm{ug}$ was completed, corresponding to the daily dose of Levothyroxine her endocrinologist prescribed $(112 \mathrm{ug})$. The patient was monitored at baseline, between each dose and up to 3 hours after the procedure was completed. There were no incidents such as urticaria, angioedema, or others. On the second day, the patient received a single-full dose of $112 \mathrm{ug}$ on an empty stomach. The medication was successfully tolerated and she was discharged. Thereafter, she tolerates daily Levothyroxine.

(Rev Med Chile 2018; 146: 394-398)

Key words: Drug Hypersensitivity; Hypersensitivity; Thyroxine.
E 1 cáncer de tiroides es una neoplasia infrecuente y corresponde a 1-2\% de todas las neoplasias reportadas ${ }^{1}$. La tiroidectomía total está recomendada en pacientes con tumores de 4 centímetros o más, o en aquellos con alguna característica de mayor extensión ${ }^{2}$, dejando a los pacientes dependientes del reemplazo hormonal exógeno con levotiroxina ${ }^{3}$. Esta prohormona sintetizada en la tiroides circula en la sangre asociada en $99 \%$ a proteínas de transferencia ${ }^{4,5}$ y su vida media en un paciente eutiroideo es de aproximadamente 7 días. En ausencia de tiroides, la levotiroxina exógena es convertida eficientemente a triyodotironina (T3), la hormona activa, manteniendo niveles de T3 estables postadministración. La liotironina o T3 exógena tiene una vida media de aproximadamente 19 h, y su concentración sérica puede alcanzar un aumento de hasta $600 \%$ después de la administración, haciéndola una alternativa menos apropiada para el reemplazo hormonal ${ }^{5}$.

Las hormonas tiroideas tienen un rol crítico en el desarrollo y función de virtualmente todos los sistemas fisiológicos. Además, se cree que el reemplazo hormonal posterior a tiroidectomía por cáncer inhibiría el crecimiento tumoral en forma indirecta por retroalimentación negativa sobre la secreción de $\mathrm{TSH}^{5}$. El riesgo de no tratar a los pacientes con hipotiroidismo es el coma mixedematoso, una emergencia endocrina caracterizada por alteración de conciencia e hipotermia con otros signos clínicos de hipotiroidismo severo ${ }^{6}$, 
que pone al paciente en riesgo vital de no mediar tratamiento rápido.

El tratamiento de reemplazo hormonal con levotiroxina es ampliamente utilizado y las reacciones de hipersensibilidad son infrecuentes. Se manifiestan principalmente como urticaria, angioedema, exantema eccematoso, fiebre y disfunción hepática ${ }^{7}$.

La levotiroxina es el tratamiento de elección para el manejo a largo plazo del hipotiroidismo ${ }^{5}$. La liotironina tiene la misma estructura química, salvo por un átomo de yodo menos, por lo cual, en la actualidad, no existe alternativa terapéutica para los pacientes alérgicos. Considerando la necesidad rápida e imperativa de usar levotiroxina, el único tratamiento existente para estos pacientes es la desensibilización.

La desensibilización medicamentosa está indicada para reacciones de perfil inmediato, anafilácticas, con diferentes grupos de fármacos: antibióticos, antiinflamatorios, suplementos vitamínicos, hormonas, fármacos antineoplásicos, agentes biológicos y otros, y debe ser realizada con el paciente hospitalizado, monitorizado, en un área con personal entrenado en el manejo de reacciones alérgicas severas ${ }^{8-11}$.

\section{Caso clínico}

Se trata de una paciente de 39 años, tiroidectomizada total por cáncer papilar de tiroides, que consultó en policlínico de Inmunología de nuestro hospital 7 meses después, con antecedente de haber presentado varios episodios de edema labial, palpebral y lingual, además de dolor abdominal, hasta 60 min después de tomar diferentes formulaciones comerciales de levotiroxina y, además, preparados magistrales de levotiroxina que contenían solamente talco en forma de silicato de Mg como excipiente. Todos los episodios habían respondido total o parcialmente a corticoides sistémicos y antihistamínicos.

Sus exámenes mostraban un hipotiroidismo grave, con hormona tiroestimulante (TSH) de $117 \mathrm{uUI} / \mathrm{mL}$ y tetrayodotironina (T4) libre de $0,15 \mathrm{ng} / \mathrm{dl}$, y anticuerpos antitiroglobulinas en rango normal. Los niveles de triyodotironina eran normales $(1,8 \mathrm{ng} / \mathrm{ml})$, lo cual es frecuente en casos de hipotiroidismo, ya que T3 proviene mayormente de la degradación periférica de T4 y no de la glándula tiroidea. La paciente presentaba síntomas y signos clínicos de bradicardia, piel seca, facies mixedematosa, caída del cabello y de las cejas, lentitud psicomotora, somnolencia, y se consideró una urgencia su estudio y tratamiento alergológico. Fue evaluada, además, por el equipo de Endocrinología, que consideró que la paciente tenía indicación absoluta de recibir periódicamente levotiroxina, y se le indicó una dosis diaria de $112 \mathrm{ug}$, la que se transformó en la dosis a alcanzar en un protocolo de desensibilización.

Se desarrolló protocolo de pruebas cutáneas, incluyendo los excipientes puros (almidón de maíz, gelatina y talco) de las principales formulaciones comerciales, salvo croscarmelosa, la que no pudimos conseguir en su forma pura con ese fin (Tabla 1). Como se trataba de presentaciones orales, solamente pudimos efectuar prick test y no intradermorreacciones.

Los resultados de los prick test resultaron negativos, tanto a levotiroxina como a excipientes, pero clínicamente la historia era fuertemente sugerente de reacciones inmediatas con el uso de diferentes formas de levotiroxina, por lo cual se le planteó a la paciente y a su familia su hospitalización en Unidad de Intermedio Médico, para efectuar protocolo de desensibilización por vía oral con levotiroxina en presentación magistral, que contenía solamente talco como excipiente. El protocolo de desensibilización se realizó previa firma de consentimiento informado.

Se revisó la literatura, donde existen escasos casos descritos de alergia y desensibilización a levotiroxina y optamos por seguir la pauta del

Tabla 1. Prick test con presentaciones comercial y magistral de levotiroxina y excipientes

\begin{tabular}{|lc|}
\hline Sustancia & $\begin{array}{c}\text { Prick test } \\
\text { (mm pápula) }\end{array}$ \\
\hline Control positivo (histamina $10 \mathrm{mg} / \mathrm{ml}^{\text {(mETI }}{ }^{\circledR}$ & $5 \mathrm{~mm}$ \\
\hline Control negativo (suero fisiológico) LETI $^{\circledR}$ & $0 \mathrm{~mm}$ \\
\hline Almidón de maíz & $0 \mathrm{~mm}$ \\
\hline Gelatina & $0 \mathrm{~mm}$ \\
\hline Talco (silicato de Mg) & $0 \mathrm{~mm}$ \\
\hline Celulosa & $0 \mathrm{~mm}$ \\
\hline Eutirox ${ }^{\circledR} 20 \mathrm{mg} / \mathrm{ml}$ & $0 \mathrm{~mm}$ \\
\hline Levotiroxina magistral en talco $100 \mathrm{mg} / \mathrm{ml}$ & $0 \mathrm{~mm}$ \\
\hline
\end{tabular}


Tabla 2. Protocolo de desensibilización a levotiroxina*. Día 1

\begin{tabular}{|cll|}
\hline Paso & Dosis/sustancia & Dosis acumulada levotiroxina \\
\hline 1 & Placebo (suero fisiológico) & \\
\hline 2 & $0,01 \mathrm{mcg} /$ levotiroxina & $0,01 \mathrm{mcg}$ \\
\hline 3 & $0,02 \mathrm{mcg} /$ levotiroxina & $0,03 \mathrm{mcg}$ \\
\hline 4 & $0,04 \mathrm{mcg} /$ levotiroxina & $0,07 \mathrm{mcg}$ \\
\hline 6 & $0,08 \mathrm{mcg} /$ levotiroxina & $0,15 \mathrm{mcg}$ \\
\hline 7 & $0,16 \mathrm{mcg} /$ levotiroxina & $0,31 \mathrm{mcg}$ \\
\hline 8 & $0,32 \mathrm{mcg} /$ levotiroxina & $0,63 \mathrm{mcg}$ \\
\hline 9 & $0,64 \mathrm{mcg} /$ levotiroxina & $1,27 \mathrm{mcg}$ \\
\hline 10 & $1,28 \mathrm{mcg} /$ levotiroxina & $2,55 \mathrm{mcg}$ \\
\hline 11 & $2,56 \mathrm{mcg} /$ levotiroxina & $5,11 \mathrm{mcg}$ \\
\hline 12 & $5,12 \mathrm{mcg} /$ levotiroxina & $10,23 \mathrm{mcg}$ \\
\hline 13 & $10,24 \mathrm{mcg} /$ levotiroxina & $20,47 \mathrm{mcg}$ \\
\hline 14 & $20,48 \mathrm{mcg} /$ levotiroxina & $40,95 \mathrm{mcg}$ \\
\hline 15 & $30 \mathrm{mcg} /$ levotiroxina & $70,95 \mathrm{mcg}$ \\
\hline
\end{tabular}

*Entre los pasos 2 y 10 se utilizó levotiroxina en solución (magistral), con las diluciones correspondientes en suero fisiológico. Entre los pasos 11 y 15 se utilizó levotiroxina en cápsulas (preparación magistral). Las respectivas dosis fueron administradas cada $15 \mathrm{~min}$, con control de la paciente basalmente, entre cada dosis y luego 15, 45 y 120 min después de terminado el procedimiento.

grupo de Fevzi et al. de Ankara, modificada ${ }^{7}$, partiendo con dosis de $0,01 \mathrm{ug}$, las que se doblaron cada $15 \mathrm{~min}$, hasta llegar a la dosis acumulada de $111,95 \mathrm{ug}$ a las $3 \mathrm{~h}$ y media de haberse iniciado el procedimiento, sin incidentes alérgicos, el primer día de nuestra intervención.

En agosto de 2016, se efectuó el procedimiento en ayunas, con vía venosa permeable y paciente monitorizada, con control de signos vitales y flujometría basalmente, entre cada dosis y 15, 45 y 120 min después de finalizar el procedimiento ( $\mathrm{Ta}-$ bla 2). La primera dosis administrada fue placebo.

El segundo día de hospitalización la paciente recibió en ayunas $(7: 30 \mathrm{~h})$, la dosis completa de 112 ug en una sola oportunidad, sin incidentes alérgicos y siendo controlada durante toda la mañana, y fue dada de alta a mediodía, tolerando hasta ahora la dosis de 112 ug diarios de levotiroxina indicada por su tratante.

Se utilizaron preparados magistrales de levotiroxina en solución, para las dosis más bajas, y posteriormente en cápsulas.
Dos meses después de la desensibilización, la paciente estaba clínicamente mejor, con valores de TSH de $0,94 \mathrm{uUI} / \mathrm{mL}$, T4 libre $1,7 \mathrm{ng} / \mathrm{dl}$, tiroglobulina $0,1 \mathrm{ng} / \mathrm{mL}$ con anticuerpos antitiroglobulina en rangos normales.

\section{Discusión}

La sustitución hormonal exógena con levotiroxina o liotironina, constituye el único tratamiento del hipotiroidismo y es tal su relevancia, que su no uso implica un riesgo vital para el enfermo que padece esta enfermedad. Una de las únicas contraindicaciones para su uso es la alergia al medicamento.

En la literatura, existen escasos reportes de alergia a la levotiroxina ${ }^{7,12,13,14,15}$ y ninguno, hasta nuestro conocimiento, para liotironina.

Es inusual que sustancias biológicas administradas por vía exógena produzcan reacciones alérgicas. Se ha descrito alergia a insulina y heparina $y$, en esos casos, las opciones terapéuticas son la 
sustitución del medicamento por otro similar o bien la desensibilización a ellos ${ }^{4}$. Considerando que en nuestro país no existe alternativa terapéutica para la levotiroxina, la única opción fue la desensibilización de la paciente.

El primer protocolo de desensibilización a levotiroxina descrito en la literatura fue el de Nugent ${ }^{13}$, en octubre de 2003. Ellos se basaron en el modelo utilizado para la desensibilización a penicilina, comenzando con $0,00005 \mathrm{mcg}$ de levotiroxina por vía endovenosa y aumentando paulatinamente la dosis con intervalos de 15$30 \mathrm{~min}(0,001 \mathrm{mcg}, 001 \mathrm{mcg}, 0,025 \mathrm{mcg}, 0,005$ $\mathrm{mcg}, 0,1 \mathrm{mcg}, 0,25 \mathrm{mcg}, 0,5 \mathrm{mcg}, 1 \mathrm{mcg}, 5 \mathrm{mcg}$, $15 \mathrm{mcg}$ y $30 \mathrm{mcg}$ ) hasta llegar a $50 \mathrm{mcg}$, con evolución favorable.

Posteriormente, el grupo español de Sala et $\mathrm{al}^{15}$ adaptó el protocolo de Nugent, aumentando la dosis inicial $(0,0002 \mathrm{mcg})$ y acercando los intervalos de dosis a 15 min entre cada uno $(0,0002$ $\mathrm{mcg}, 0,0004 \mathrm{mcg}, 0,004 \mathrm{mcg}, 0,02 \mathrm{mcg}, 0,04 \mathrm{mcg}$, $0,1 \mathrm{mcg}, 0,2 \mathrm{mcg}, 4 \mathrm{mcg}, 1 \mathrm{mcg}, 5 \mathrm{mcg}, 15 \mathrm{mcg}$, $30 \mathrm{mcg}$ y $50 \mathrm{mcg}$ ), con resultado exitoso.

Considerando que los pacientes habitualmente utilizan levotiroxina terapéutica mediante vía oral, es que el grupo turco de Fevzi y colaboradores ${ }^{7}$ creó el primer protocolo de desensibilización a levotiroxina por vía oral, que comenzaba con 0,01 mcg como primera dosis e iba doblando sus concentraciones cada $30 \mathrm{~min}$, hasta llegar a una dosis acumulada de $81,91 \mathrm{mcg}$.

Comunicamos el primer caso en Chile, hasta nuestro conocimiento, de alergia a la levotiroxina y su exitosa desensibilización posterior. Nuestro grupo se basó en el protocolo de desensibilización por vía oral del grupo de Fevzi ${ }^{7}$, ya que en nuestro país, solo contamos con levotiroxina de uso oral. Ajustamos el protocolo hasta llegar a una dosis acumulada de 111,95 mcg, tal como fue aconsejado por el equipo de Endocrinología de nuestro hospital. Considerando que los protocolos reportados en la literatura mediante vía endovenosa, que poseen mayor riesgo de producir reacciones sistémicas, fueron administrando dosis cada $15 \mathrm{~min}^{9,11}$, fue que decidimos emular el modelo y fuimos incrementando las dosis cada $15 \mathrm{~min}$, sin reacciones adversas, con una respuesta favorable. Posteriormente, la paciente siguió tolerando la dosis completa a diario, con cese de todos los síntomas que le alteraban de forma significativa su calidad de vida.
Aunque el hipotiroidismo es una patología muy frecuente en nuestra población, la alergia a la levotiroxina es infrecuente ${ }^{12,13,14}$. Sin embargo, existe y es una reacción que debemos conocer. En los casos en que ocurre, recomendamos la rápida derivación para la realización de desensibilización, que es el único tratamiento que permite la sobrevida de estos pacientes.

\section{Referencias}

1. Liberman C. Prevalencia e incidencia de los principales trastornos endocrinos y metabólicos. Rev Med Clin Condes 2013; 24 (5): 735-41.

2. Mitchell AL, Gandhi A, Scott-Coombes D, Perros P. Management of thyroid cancer: United Kingdom National Multidisciplinary Guidelines. J Laryngol Otol 2016; 130 (S2): S150-60.

3. Jonklaas J. Update on the treatment of hypothyroidism. Curr Opin Oncol 2016; 28 (1): 18-25.

4. Mondal S, Raja K, Schweizer U, Mugesh G. Chemistry and Biology in the Biosynthesis and Action of Thyroid Hormones. Angew Chem Int Ed Engl 2016; 55 (27): 7606-30.

5. Hannoush ZC, Weiss RE. Thyroid hormone replacement in patients following thyroidectomy for thyroid cancer. Rambam Maimonides Med J 2016; 7(1).

6. Gish DC, Loynd RT, Melnick S, Nazir R. Myxoedema coma: a forgotten presentation of extreme hypothyroidism. BMJ Case Rep. Published online: [28 Jun 2016].

7. Fezvi D, Mustafa G, Ozgur K, Cetin T, Abdullah B, Sait Yet al. Successful oral desensitization to levothyroxine. Ann Allegy Asthma Immunol 2013; 111 (2): 146-7.

8. Audicana Berasategui MT, Tornero Molina P, Ortega Rodríguez NR, Rosado Ingelmo A. Prevención y tratamiento de las reacciones de hipersensibilidad inducidas por los fármacos. Desensibilización. En Tratado de Alergología, Segunda Edición, 2016, I. Dávila, I. Jáuregui, J. Olaguibel, J. Zubeldia, Ed. Ergon, Majadahonda, Madrid, España. P. 1465-93.

9. Alvarez-Cuesta E, Madrigal-Burgaleta R, Angel-Pereira D, Ureña-Tavera A, Zamora-Verduga M, López-González $\mathrm{P}$, et al. Delving into cornerstones of hypersensitivity to antineoplastic and biological agents: value of diagnostic tools prior to desensitization. Allergy 2015; 70 (7): 784-94.

10. Madrigal-Burgaleta R, Berges-Gimeno MP, Angel-Pereira D, Ferreiro-Monteagudo R, Guillen-Ponce C, Pueyo $\mathrm{C}$, et al. Hypersensitivity and desensitization to antineo- 
plastic agents: Outcomes of 189 procedures with a new short protocol and novel diagnostic tools assessment. Allergy 2013; 68: 853-61.

11. González E, Henríquez A, Losada A, Huertas B, González J, Berges $\mathrm{P}$, et al. Pyrimethamine desensitization in cerebral toxoplasmosis and AIDS. J Allergy Clin Immunol 2007; 119: Abs 261 S67.

12. Shibata H, Hayakawa H, Hirukawa M, Tadokoro K, Ogata E. Hypersensitivity caused by synthetic thyroid hormones in hypothyroid patient with Hashimoto's thyroiditis. Arch Intern Med 1986;146: 1624-5.
13. Nugent JS, Nugent AL, Whisman BA, White K, Hagan LL. Levothyroxine anaphylaxis? Vocal cord dysfunction mimicking an anaphylactic drug reaction. Ann Allergy Asthma Immunol 2003; 91 (4): 337-41.

14. Kaur S, Bhalla M, Thami GP. Subacute lichenoid eruption due to L-thyroxine overdosage. Dermatology 2003; 206: 346-7.

15. Sala A, Labrador-Horrillo M, Guilarte M, Luengo O, Rueda M, Cardona V. Immediate-type hypersensitivity reaction to levothyroxine and desensitization. Ann Allergy Asthma Immunol 2008; 100 (5): 513-4. 